\title{
ON A MODIFIED BURR XII DISTRIBUTION HAVING FLEXIBLE HAZARD RATE SHAPES
}

\author{
FARRUKH JAMAL ${ }^{1}$, CHRISTOPHE CHESNEAU ${ }^{2}$, M. ARSLAN NASIR ${ }^{3}$, ABDUS SABOOR $^{4}$, \\ EMRAH ALTUN ${ }^{5}$, M. AZAM KHAN ${ }^{6}$
}

\begin{abstract}
In this paper, we propose a new three parameter modified Burr XII distribution based on the standard Burr XII distribution and the composition technique developed by [13]. Among others, we show that this technique has the ability to significantly increase the flexibility of the former Burr XII distribution, with respect to the density and hazard rate shapes. Also, complementary theoretical aspects are studied as shapes, asymptotes, quantiles, useful expansion, moments, skewness, kurtosis, incomplete moments, moment generating function, stochastic ordering, reliability parameter and order statistics. Then, by considering the Burr XII distribution as a parametric model, a statistical inference is performed. A Monte Carlo simulation study is carried out to assess the performance of the maximum likelihood estimates. Finally, three applications to real-life data sets are presented, with models comparisons. The results are favorable for the new modified Burr XII model.
\end{abstract}

Key words : Burr XII distribution, Compounding distribution, stochastic ordering, reliability, order statistics.

AMS SUBJECT : Primary 60E05, 62E15, 62F10.

\section{INTRODUCTION}

The Burr XII distribution introduced by [7] is a well-recognized distribution in probability theory, statistics and economics, with numerous applications in health, agriculture, actuarial sciences and biology. From a mathematical point of view, the cumulative distribution function (cdf), probability density function (pdf) and hazard rate function (hrf) of the Burr XII distribution are respectively given by

$$
F_{B X I I}(x)=1-\left(1+x^{c}\right)^{-k}, \quad x, c, k>0,
$$

\footnotetext{
${ }^{1}$ Govt. S.A P/G College Dera Nawab Sahib, Bahawalpur, Pakistan. email: farrukhjamalmphil@gmail.com

${ }^{2}$ Department of Mathematics, LMNO, University of Caen-Normandie, Caen, France. email: christophe.chesneau@gmail.com

${ }^{3}$ Govt S.E College Bahawalpur, Pakistan. email: arslannasir147@gmail.com (M. A. N.)

${ }^{4}$ Kohat University of Science and Technology, Pakistan. email: saboorhangu@gmail.com

${ }^{5}$ Department of Statistics, Hacettepe University, Turkey. email: emrahaltun123@gmail.com

${ }^{6}$ Jaipuria Institute of Management, Lucknow-226 010, India. email: khanazam2808@gmail.com.
} 
and

$$
f_{B X I I}(x)=c k x^{c-1}\left(1+x^{c}\right)^{-k-1}
$$

$$
h_{B X I I}(x)=c k x^{c-1}\left(1+x^{c}\right)^{-k-1} .
$$

Despite its numerous merits, one limitation of the Burr XII distribution is to have a lack of flexibility on the hrf; it can be decreased for $c \leq 1$, it is essentially constant for $c \in(1,2)$, and it increases to reach a maximum value before decreasing for $c>2$. This aspect constitutes an obstacle for modelling certain lifetime data sets which require nonmonotonic shapes for the hrf like the bathtub shape, the unimodal (upside-down bathtub) or modified unimodal shape and many others needs various monotonic shapes (as human mortality, machine life cycles and data from some biological and medical studies...). Further details on the Burr XII distribution can be found in [7], [22], [25], [10], [19], [5], [15] and [1]. Consequently, numerous modification or generalizations of the BXII distribution, using different compounding and weighting techniques, have been suggested. See, for instance, the beta Burr XII distribution introduced by [20], the McDonald Burr XII distribution introduced by [11], the Poisson Burr XII distribution established by [16], the Marshall-Olkin extended Burr XII distribution developed by [6], the Weibull Burr XII distribution introduced by [3] and the transmuted Burr XII distribution developed by [4].

On the other side, an original modification technique has been introduced by [13] in the context of the Weibull distribution. It consists in compounding the Weibull cdf by a polyno-exponential function of the form $e^{\lambda x} x^{c}$ with $c, \lambda>0$. Hence, it is proved that the former Weibull distribution is significantly enriched by this modification, opening new perspectives of modelling. In fact, this modification are still effective for any cdf with support on $(0,+\infty)$. Based on this remark, we propose a new three parameter modified Burr XII distribution called modified Burr XII distribution (MBXII). As shown in this study, this new modification gives various kinds of shapes of the pdf and hrf, and particularly, monotonic increasing, decreasing, bathtub, unimodal and constant hrf shapes, which are not always reached for the former Burr XII distribution. From the mathematical point of view, the MBXII distribution is characterized by the cdf given by

$$
F_{M B X I I}(x)=1-\left(1+e^{\lambda x} x^{c}\right)^{-k}, \quad x>0,
$$

with $k>0, c, \lambda \geq 0$ and $\max (c, \lambda)>0$. The pdf and hazard rate functions corresponding to equation (1) are respectively given by

$$
f_{M B X I I}(x)=k e^{\lambda x} x^{c}\left[\frac{c}{x}+\lambda\right]\left(1+e^{\lambda x} x^{c}\right)^{-k-1}
$$

and

$$
h_{M B X I I}(x)=\left[\frac{c}{x}+\lambda\right] \frac{k e^{\lambda x} x^{c}}{1+e^{\lambda x} x^{c}} .
$$

To show the richness of the MBXII distribution, Table 1 lists some special cases, including well established and new distributions (with a certain potential of interest for the statistician). 
TABle 1. Special cases of the MBXII distribution.

\begin{tabular}{|l|lll|l|l|}
\hline Distribution & $c$ & $k$ & $\lambda$ & $F_{M B X I I}(x)$ & Reference \\
\hline \hline Burr XII & - & - & 0 & $1-\left(1+x^{c}\right)^{-k}$ & {$[7]$} \\
\hline Modified Log-logistic & - & 1 & - & $\frac{e^{\lambda x} x^{c}}{1+e^{\lambda x} x^{c}}$ & New* \\
\hline Log-logistic & - & 1 & 0 & $\frac{x^{c}}{1+x^{c}}$ & {$[24]$} \\
\hline Logistic & 0 & 1 & - & $\frac{e^{\lambda x}}{1+e^{\lambda x}}$ & {$[9]$} \\
\hline Lomax & 1 & - & 0 & $1-(1+x)^{-k}$ & {$[14]$} \\
\hline Modified Lomax & 1 & - & - & $1-\left(1+e^{\lambda x} x\right)^{-k}$ & New* \\
\hline Modified Logistic & 1 & 1 & - & $\frac{e^{\lambda x} x}{1+e^{\lambda x}}$ & New* \\
\hline
\end{tabular}

The paper is organized as follows. The central functions of the MBXII distribution are studied in Section 2, including shapes, asymptotes and useful expansions. In Section 3, we provide a comprehensive account of the main mathematical properties of the MBXII distribution, including moments, incomplete moments, moment generating function, mode, stochastic ordering, reliability parameter and order statistics. Statistical inference is studied in Section 4, where the estimations of the model parameters are performed by the maximum likelihood method, as well as a simulation study. Applications to three data sets of various nature are explored in Section 5. Some conclusions are given in Section 6 .

\section{ON THE MBXII FUNCTIONS}

In this section, some results on shapes, asymptotes and useful expansions are established for the MBXII cdf, i.e., $F_{M B X I I}(x)$ given by (1), the MBXII pdf, i.e., $f_{M B X I I}(x)$ given by $(2)$ and the MBXII hrf, i.e., $h_{M B X I I}(x)$ given by $(3)$. Hereafter, since it is the more realistic case in practice, for the sake of simplicity, we suppose that $c, \lambda>0$ (excluding $c=0$ or $\lambda=0)$.

2.1. Shapes and asymptotes. The critical points of the MBXII pdf are the solution of the following equation: $\partial \log \left[f_{M B X I I}(x)\right] / \partial x=0$, i.e.,

$$
\frac{c}{x}+\lambda-\frac{c}{x(c+\lambda x)}-(k+1)\left[\frac{c}{x}+\lambda\right] \frac{e^{\lambda x} x^{c}}{1+e^{\lambda x} x^{c}}=0 .
$$

Similarly, the critical points of the MBXII hrf are the solution of the following equation: 


$$
\begin{aligned}
& \partial \log \left[h_{M B X I I}(x)\right] / \partial x=0 \text {, i.e. } \\
& \frac{c}{x}+\lambda-\frac{c}{x(c+\lambda x)}-\left[\frac{c}{x}+\lambda\right] \frac{x^{c} e^{\lambda x}}{1+e^{\lambda x} x^{c}}=0 .
\end{aligned}
$$

We can examine these critical points, as well as their nature (local maximum, local minimum, inflexion points ...) by using any computational package (R, Mathematica, Matlab....).

When $x \rightarrow 0$, we have the following asymptotic results:

$$
F_{M B X I I}(x) \sim k x^{c}, \quad f_{M B X I I}(x) \sim c k x^{c-1}, \quad h_{M B X I I}(x) \sim c k x^{c-1} .
$$

Thus, $f_{M B X I I}(x)$ tends to $+\infty$ when $c<1$, to $k$ when $c=1$ and to 0 when $c>1$. The same for $h_{M B X I I}(x)$. We observe that the parameter $\lambda$ plays no role in these asymptotic results.

Similarly, when $x \rightarrow+\infty$, we have the following asymptotic results:

$$
F_{M B X I I}(x) \sim 1-e^{-\lambda k x} x^{-c k}, \quad f_{M B X I I}(x) \sim \lambda k e^{-\lambda k x} x^{-c k}, \quad h_{M B X I I}(x) \sim \lambda k .
$$

Hence, $f_{M B X I I}(x)$ tends to 0 with a polyno-exponential decay, for all the possible values of the parameters, whereas $h_{M B X I I}(x)$ tends to the constant $\lambda k$. In this case, $\lambda$ is of great importance.

Some plots of the MBXII pdf and hrf for different parametric values are shown in Figures 1 and 2, respectively.
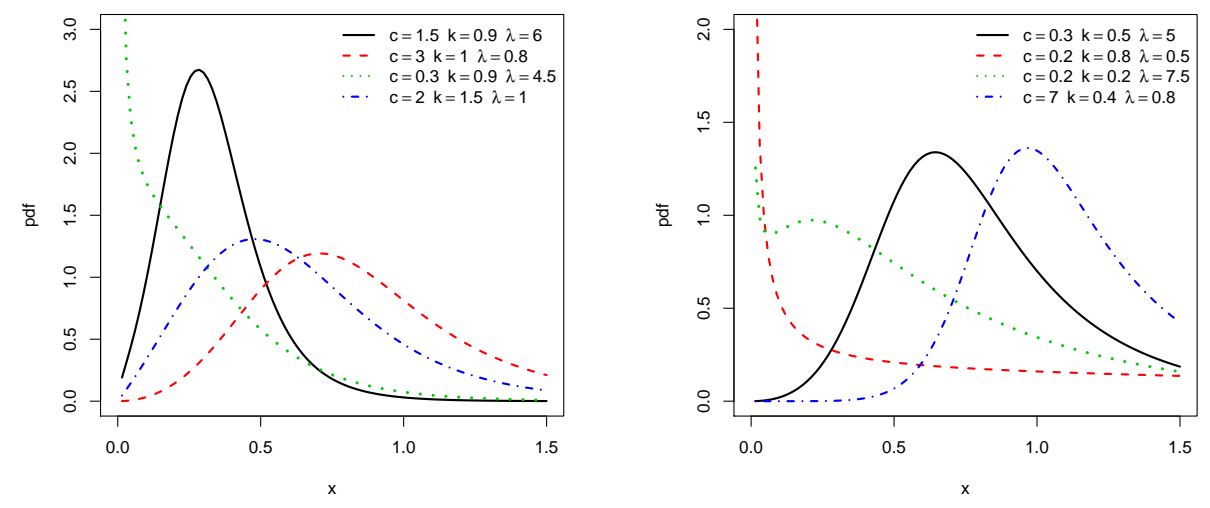

Figure 1. Plots of the MBXII pdf with different parameter values.

It is observed that the MBXII pdf has reversed J, right skewed and nearly symmetrical shapes while MBXII hrf has increasing, decreasing, upside-down bathtub and constant shapes. We thus see that the MBXII distribution offers more degree of flexibility with respect to the pdf and hrf in comparison to the former Burr XII distribution. 

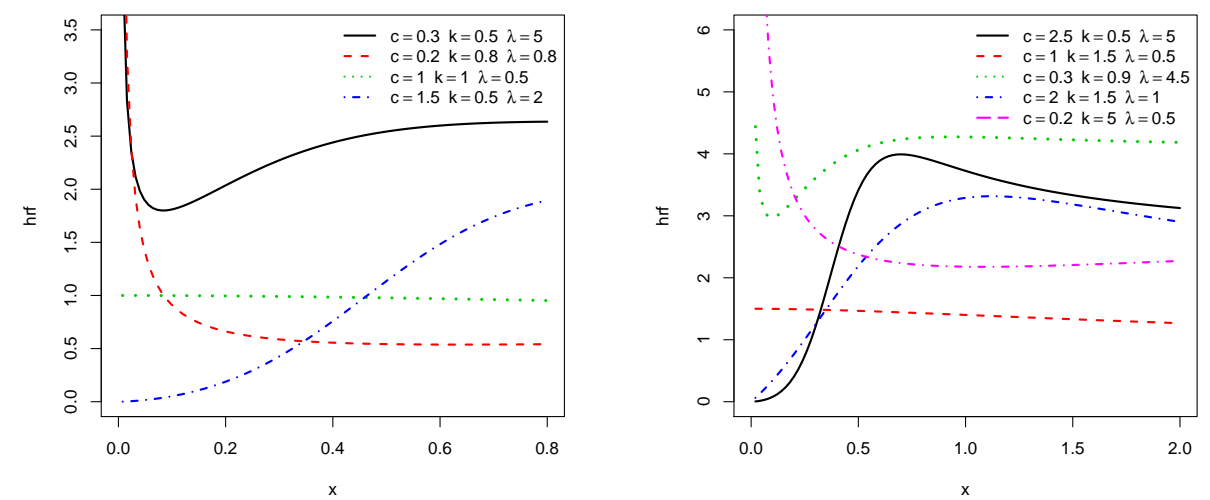

Figure 2. Plots of the MBXII hrf with different parameter values.

2.2. Useful expansion. Since $e^{\lambda x} x^{c}$ is a continuous and increasing function over $(0,+\infty)$, there exists an unique real number $x_{*}$, depending on $\lambda$ and $c$, such that $e^{\lambda x_{*}} x_{*}^{c}=1$ with $e^{\lambda x} x^{c}<1$ for $x \in\left(0, x_{*}\right)$, and $e^{\lambda x} x^{c}>1$ for $x>x_{*}$. The generalized binomial formula gives

$$
F_{M B X I I}(x)=1- \begin{cases}\sum_{j=0}^{+\infty}\left(\begin{array}{c}
-k \\
j
\end{array}\right) e^{j \lambda x} x^{c j} & \text { si } x \in\left(0, x_{*}\right), \\
2^{-k} & \text { si } x=x_{*}, \\
\sum_{j=0}^{+\infty}\left(\begin{array}{c}
-k \\
j
\end{array}\right) e^{-(j+k) \lambda x} x^{-c(j+k)} & \text { si } x>x_{*},\end{cases}
$$

where $\left(\begin{array}{l}b \\ a\end{array}\right)$ denotes the binomial coefficients, i.e., $\left(\begin{array}{l}b \\ a\end{array}\right)=b(b-1) \ldots(b-a+1) / a$ !.

Therefore, almost surely, we can express $f_{M B X I I}(x)$ as

$$
f_{M B X I I}(x)= \begin{cases}-\sum_{j=0}^{+\infty}\left(\begin{array}{c}
-k \\
j
\end{array}\right) j\left[\frac{c}{x}+\lambda\right] e^{j \lambda x} x^{c j} & \text { si } x \in\left(0, x_{*}\right), \\
\sum_{j=0}^{+\infty}\left(\begin{array}{c}
-k \\
j
\end{array}\right)(j+k)\left[\frac{c}{x}+\lambda\right] e^{-(j+k) \lambda x} x^{-c(j+k)} & \text { si } x>x_{*} .\end{cases}
$$

This linear expansion will be useful to express several probabilistic quantities defined as integrals of the form $\int_{-\infty}^{+\infty} w(x) f_{M B X I I}(x) d x$, where $w(x)$ denotes any function (such that the integral exists). 


\section{Statistical PROPERTies}

The quantiles, moments, skewness, kurtosis, incomplete moments, moment generating function, stochastic ordering, reliability coefficient and order statistics are obtained in this section.

3.1. Quantiles. The quantile function, denoted by $Q_{M B X I I}(y)$, satisfies the equation: $F_{M B X I I}\left[Q_{M B X I I}(y)\right]=y$ for any $y \in(0,1)$. After some algebra, we found

$$
Q_{M B X I I}(y)=\frac{c}{\lambda} W\left(\frac{\lambda}{c}\left[(1-y)^{-1 / k}-1\right]^{1 / c}\right), \quad y \in(0,1),
$$

where $W(x)$ tis the so-called product log function, i.e., the function which is given by the principal solution $w$ for $w e^{w}=x$.

For given $y, c, k$ and $\lambda, Q(y)$ can be computed numerically via the use of any standard softwares, as R, Mapple, Mathematica.... All the special quantiles can be derived, as the median given by $M_{e d}=Q_{M B X I I}(0.5)$. Also, for a random variable $U$ following the uniform distribution on $(0,1)$, the random variable $X$ defined by $X=Q_{M B X I I}(U)$ follows the MBXII distribution. This is useful to generate random numbers distributed from the MBXII distribution.

3.2. Moments. The $r^{t h}$ moment of the MBXII distribution is given by

$$
\mu_{r}^{\prime}=\int_{-\infty}^{+\infty} x^{r} f_{M B X I I}(x) d x=\int_{0}^{+\infty} k x^{r+c} e^{\lambda x}\left[\frac{c}{x}+\lambda\right]\left(1+e^{\lambda x} x^{c}\right)^{-k-1} d x
$$

Since $x^{r} f_{M B X I I}(x) \sim c k x^{r+c-1}$ when $x \rightarrow 0$ and $x^{r} f_{M B X I I}(x) \sim \lambda k e^{-\lambda k x} x^{r-c k}$ when $x \rightarrow$ $+\infty$, using the Riemann integral, we prove that $\mu_{r}^{\prime}$ exists. To the best of our knowledge, there is no close form for $\mu_{r}^{\prime}$ (even with the standard change of variable $x=Q_{M B X I I}(y)$ ). However, for given $c, k$ and $\lambda$, it can be evaluated numerically with standard softwares.

The following result provides lower and upper bounds for $\mu_{r}^{\prime}$, under some conditions on the parameters. This allows to have a quick evaluation of the possible values for $\mu_{r}^{\prime}$.

Proposition 1. Let $\Gamma(a)$ be the Gamma function defined by $\Gamma(a)=\int_{0}^{+\infty} t^{a-1} e^{-t} d t$, with $a>0$. Then, for $k c<r$, we have the following upper bound:

$$
\mu_{r}^{\prime} \leq \frac{r}{(\lambda k)^{r-k c}} \Gamma(r-k c) .
$$

On the other hand, without restriction on the parameters, we have the following lower bound: for any $x_{0}>0$, we have

$$
\mu_{r}^{\prime} \geq x_{0}^{r}\left(1+e^{\lambda x_{0}} x_{0}^{c}\right)^{-k} .
$$


Proof. Using the inequalities: $\left(1+e^{\lambda x} x^{c}\right)^{-k-1} \leq e^{-\lambda(k+1) x} x^{-c(k+1)}$, some changes of variables and the equality: $\Gamma(\alpha+1)=\alpha \Gamma(\alpha)$, we have

$$
\begin{aligned}
\mu_{r}^{\prime} & \leq \int_{0}^{+\infty} k x^{r-k c} e^{-\lambda k x}\left[\frac{c}{x}+\lambda\right] d x \\
& =k c \int_{0}^{+\infty} x^{r-k c-1} e^{-\lambda k x} d x+\lambda k \int_{0}^{+\infty} x^{r-k c} e^{-\lambda k x} d x \\
& =\frac{k c}{(\lambda k)^{r-k c}} \Gamma(r-k c)+\frac{\lambda k}{(\lambda k)^{r-k c+1}} \Gamma(r-k c+1) \\
& =\frac{1}{(\lambda k)^{r-k c}}[k c+r-k c] \Gamma(r-k c)=\frac{r}{(\lambda k)^{r-k c}} \Gamma(r-k c) .
\end{aligned}
$$

The upper bound is proved. For the lower bound, the Markov inequality for positive random variables implies that, for any $x_{0}>0$,

$$
\mu_{r}^{\prime} \geq x_{0}^{r}\left[1-F_{M B X I I}\left(x_{0}\right)\right]=x_{0}^{r}\left(1+e^{\lambda x_{0}} x_{0}^{c}\right)^{-k}
$$

This ends the proof of Proposition 1.

Now, let us present an expansion series for $\mu_{r}^{\prime}$. First of all, we introduce the two following integral functions:

$$
\mathcal{I}(a, x)=\int_{0}^{x} t^{a-1} e^{t} d t, \quad \Gamma(b, x)=\int_{x}^{+\infty} t^{b-1} e^{-t} d t
$$

with $a>0, x>0$ and $b \in \mathbb{R}$. Note that $\Gamma(b, x)$ is the so-called upper incomplete gamma function (which exists for $b \in \mathbb{R}$ if $x>0$ ), whereas $\mathcal{I}(a, x)$ is not a special integral to the best of our knowledge. One can notice that, if $a$ is a positive integer, $\mathcal{I}(a, x)$ can be calculated via $a-1$ integration by parts. More generally, using the exponential seres expansions, the integral $\mathcal{I}(a, x)$ can be expressed as

$$
\mathcal{I}(a, x)=x^{a} \sum_{k=0}^{+\infty} \frac{x^{k}}{k !(k+a)} .
$$


With these notations, by virtue of the expansion (4), we have

$$
\begin{aligned}
\mu_{r}^{\prime} & =-\sum_{j=0}^{+\infty}\left(\begin{array}{c}
-k \\
j
\end{array}\right)\left[c j \int_{0}^{x_{*}} x^{c j+r-1} e^{j \lambda x} d x+\lambda j \int_{0}^{x_{*}} x^{c j+r} e^{j \lambda x} d x\right. \\
& -\left\{c(j+k) \int_{x_{*}}^{+\infty} x^{-c(j+k)+r-1} e^{-(j+k) \lambda x} d x\right. \\
& \left.\left.+\lambda(j+k) \int_{x_{*}}^{+\infty} x^{-c(j+k)+r} e^{-(j+k) \lambda x} d x\right\}\right] \\
& =-\sum_{j=0}^{+\infty}\left(\begin{array}{c}
-k \\
j
\end{array}\right)\left[\frac{1}{(\lambda j)^{c j+r}}\left\{c j \mathcal{I}\left(c j+r, j \lambda x_{*}\right)+\mathcal{I}\left(c j+r+1, j \lambda x_{*}\right)\right\}\right. \\
& -\frac{1}{[(j+k) \lambda]^{-c(j+k)+r}}\left\{c(j+k) \Gamma\left(-c(j+k)+r,(j+k) \lambda x_{*}\right)\right. \\
& \left.+\Gamma\left(-c(j+k)+r+1,(j+k) \lambda x_{*}\right)\right\}
\end{aligned}
$$

In particular, the mean and variance of the MBXII distribution are respectively given by $\mu=\mu_{1}^{\prime}$ and $\sigma^{2}=\mu_{2}^{\prime}-\left(\mu_{1}^{\prime}\right)^{2}$. Plots of these quantities for $c=2$ are shown in Figure 3 . Based on these plots, we observe that, when the parameters $k$ and $\lambda$ increase, the mean decreases, and if $k$ increases, the variance decreases and parameter $k$ has more significant effect on variance than parameter $\lambda$.

Also, the $r^{t h}$ central moment of the MBXII distribution are defined by $\mu_{r}=\mathbb{E}\left[\left(X-\mu_{1}^{\prime}\right)^{r}\right]$. It follows immediately from the binomial formula, i.e.,

$$
\mu_{r}=\sum_{k=0}^{r}\left(\begin{array}{l}
r \\
k
\end{array}\right)(-1)^{k}\left(\mu_{1}^{\prime}\right)^{k} \mu_{r-k}^{\prime},
$$

The fourth central moments allow us to determine standard measures as the coefficients of skewness and kurtosis, respectively given by

$$
S=\frac{\mu_{3}}{\mu_{2}^{3 / 2}}, \quad K=\frac{\mu_{4}}{\mu_{2}^{2}} .
$$

Plots of the skewness and kurtosis of the MBXII distribution for $c=2$ are shown in Figure 4. We see that, when $k$ and $\lambda$ increase, the skewness and kurtosis decrease. Moreover, parameter $k$ has more significant effect on skewness and kurtosis measures than parameter $\lambda$.

Table 2 shows some moments, skewness and kurtosis for the MBXII distribution for selected values of the parameters. 
TABLE 2. Some moments, skewness and kurtosis of $X$ for MBXII distribution for the following selected parameters values in order $(c, k, \lambda) ;(\mathrm{i})$ : $(1,2,2)$, (ii): $(2,1,2)$, (iii): $(2,2,1),($ iv $):(0.1,2,1),(\mathrm{v}):(0.1,0.2,0.1),(\mathrm{vi})$ : $(13,10,2)$ and (vi): $(15,1.6,0.5)$.

\begin{tabular}{|c|c|c|c|c|c|c|c|}
\hline & (i) & (ii) & (iii) & (iv) & (v) & (vi) & (vii) \\
\hline \hline$\mu_{1}^{\prime}$ & 0.3027 & 0.6239 & 0.5440 & 0.2129 & 11.8971 & 0.7227 & 0.9298 \\
\hline$\mu_{2}^{\prime}$ & 0.1484 & 0.5047 & 0.3828 & 0.2566 & 369.7102 & 0.5264 & 0.8734 \\
\hline$\mu_{3}^{\prime}$ & 0.0974 & 0.5156 & 0.3312 & 0.4412 & 13236.64 & 0.3861 & 0.8284 \\
\hline$\mu_{4}^{\prime}$ & 0.0801 & 0.6611 & 0.3452 & 0.9654 & 512453.1 & 0.2850 & 0.7933 \\
\hline$\sigma^{2}$ & 0.0567 & 0.1154 & 0.0869 & 0.2112 & 228.1670 & 0.0040 & 0.0087 \\
\hline$S$ & 1.3428 & 1.4403 & 1.1065 & 3.0541 & 0.9891 & -0.6905 & 0.0013 \\
\hline$K$ & 5.7336 & 7.3997 & 5.5067 & 14.6334 & 2.6203 & 3.8743 & 3.8292 \\
\hline
\end{tabular}

Mean

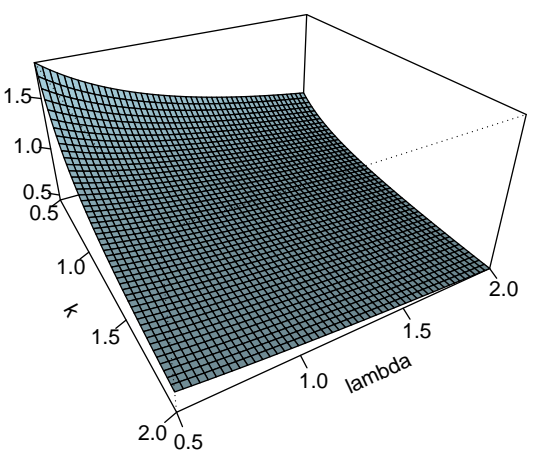

Variance

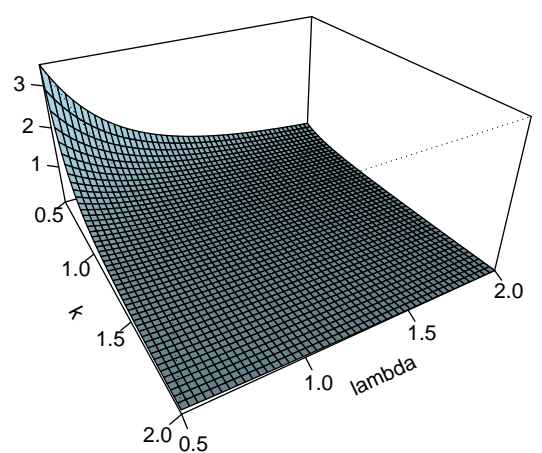

Figure 3. Plots of the mean and variance of the MBXII distribution for $c=2$

3.3. Incomplete moments. The $s^{\text {th }}$ incomplete moment of the MBXII distribution is given by

$$
\begin{aligned}
T_{s}^{\prime}(t) & =\int_{-\infty}^{t} x^{s} f_{M B X I I}(x) d x=\int_{0}^{t} k x^{s+c} e^{\lambda x}\left[\frac{c}{x}+\lambda\right]\left(1+e^{\lambda x} x^{c}\right)^{-k-1} d x, \\
t & \geq 0
\end{aligned}
$$



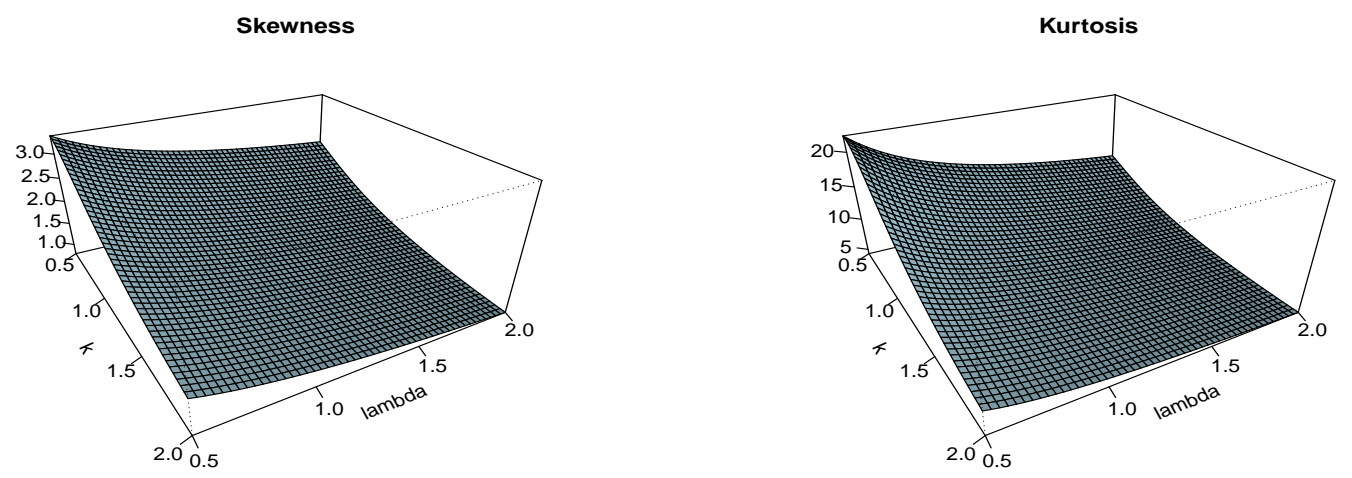

Figure 4. Plots of the skewness and kurtosis of the MBXII distribution for $c=2$

We can express $T_{s}^{\prime}(t)$ in a similar manner to $\mu_{r}^{\prime}$, by distinguishing the cases $t \leq x_{*}$ and $t>x_{*}$. In the case $t \leq x_{*}$, we have

$$
T_{s}^{\prime}(t)=-\sum_{j=0}^{+\infty}\left(\begin{array}{c}
-k \\
j
\end{array}\right) \frac{1}{(\lambda j)^{c j+s}}\{c j \mathcal{I}(c j+s, j \lambda t)+\mathcal{I}(c j+s+1, j \lambda t)\} .
$$

In the case $t>x_{*}$, we have

$$
\begin{aligned}
T_{s}^{\prime}(t) & =-\sum_{j=0}^{+\infty}\left(\begin{array}{c}
-k \\
j
\end{array}\right)\left[\frac{1}{(\lambda j)^{c j+s}}\left\{c j \mathcal{I}\left(c j+s, j \lambda x_{*}\right)+\mathcal{I}\left(c j+s+1, j \lambda x_{*}\right)\right\}\right. \\
& -\frac{1}{[(j+k) \lambda]^{-c(j+k)+s}} \times \\
& \left\{c(j+k)\left[\Gamma\left(-c(j+k)+s,(j+k) \lambda x_{*}\right)-\Gamma(-c(j+k)+s,(j+k) \lambda t)\right]\right. \\
& \left.\left.+\Gamma\left(-c(j+k)+s+1,(j+k) \lambda x_{*}\right)-\Gamma(-c(j+k)+s+1,(j+k) \lambda t)\right\}\right] .
\end{aligned}
$$

For instance, the first incomplete moment given by $T_{1}^{\prime}(t)$ is a central tool to express the mean deviations about the mean, the mean deviation about the median, the Bonferroni and Lorenz curves and the mean residual life and mean reversed residual life functions. 
More generally, the $s^{\text {th }}$ incomplete moment is involved in various important as the $s^{\text {th }}$ moment of the residual life given by

$$
\begin{aligned}
Z_{s}(t) & =\frac{1}{1-F_{M B X I I}(t)} \int_{t}^{+\infty}(x-t)^{s} f_{M B X I I}(x) d x \\
& =\left(1+e^{\lambda t} t^{c}\right)^{k} \sum_{j=0}^{s}\left(\begin{array}{l}
s \\
j
\end{array}\right)(-1)^{s-j} t^{s-j}\left[\mu_{j}^{\prime}-T_{j}^{\prime}(t)\right], \quad t \geq 0 .
\end{aligned}
$$

A similar development exists for the reversed moment of the residual life, i.e.,

$$
\begin{aligned}
W_{s}(t) & =\frac{1}{F_{M B X I I}(t)} \int_{0}^{t}(t-x)^{s} f_{M B X I I}(x) d x \\
& =\frac{1}{1-\left(1+e^{\lambda t} t^{c}\right)^{-k}} \sum_{j=0}^{s}\left(\begin{array}{l}
s \\
j
\end{array}\right) t^{s-j}(-1)^{j} T_{j}^{\prime}(t), \quad t \geq 0 .
\end{aligned}
$$

3.4. Moment generating function. The moment generating function of the MBXII distribution is given by

$$
\begin{aligned}
M_{0}(t) & =\int_{-\infty}^{+\infty} e^{t x} f_{M B X I I}(x) d x \\
& =\int_{0}^{+\infty} k x^{c} e^{(t+\lambda) x}\left[\frac{c}{x}+\lambda\right]\left(1+e^{\lambda x} x^{c}\right)^{-k-1} d x .
\end{aligned}
$$

This function is well defined for $t<\lambda k$ (the negative values are possible). Again, we can express $M_{0}(t)$ in a similar manner to $\mu_{r}^{\prime}$. We have

$$
\begin{aligned}
M_{0}(t) & =-\sum_{j=0}^{+\infty}\left(\begin{array}{c}
-k \\
j
\end{array}\right)\left[c j \int_{0}^{x_{*}} x^{c j-1} e^{(t+j \lambda) x} d x+\lambda j \int_{0}^{x_{*}} x^{c j} e^{(t+j \lambda) x} d x\right. \\
& -\left\{c(j+k) \int_{x_{*}}^{+\infty} x^{-c(j+k)-1} e^{-[(j+k) \lambda-t] x} d x\right. \\
& \left.\left.+\lambda(j+k) \int_{x_{*}}^{+\infty} x^{-c(j+k)} e^{-[(j+k) \lambda-t] x} d x\right\}\right] \\
& =-\sum_{j=0}^{+\infty}\left(\begin{array}{c}
-k \\
j
\end{array}\right)\left[\frac{1}{(t+j \lambda)^{c j}}\left\{c j \mathcal{I}\left(c j,(t+j \lambda) x_{*}\right)+\frac{\lambda j}{t+j \lambda} \mathcal{I}\left(c j+1,(t+j \lambda) x_{*}\right)\right\}\right. \\
& -\frac{1}{[(j+k) \lambda-t]-c(j+k)}\left\{c(j+k) \Gamma\left(-c(j+k),[(j+k) \lambda-t] x_{*}\right)\right. \\
+ & \left.\left.\frac{\lambda(j+k)}{(j+k) \lambda-t} \Gamma\left(-c(j+k)+1,[(j+k) \lambda-t] x_{*}\right)\right\}\right]
\end{aligned}
$$


As usual, we can recover the $r^{t h}$ moment by using the formula: $\mu_{r}^{\prime}=M_{0}^{(r)}(0)$.

3.5. Stochastic ordering. We now prove a result on the stochastic ordering involving the MBXII distribution with $c$ and $\lambda$ as common parameters. Further details on stochastic ordering can be found in [23].

Proposition 2. Let $X$ be a random variable following the MBXII distribution with parameters $c, k_{1}$ and $\lambda$, i.e., with the pdf given by

$$
f_{M B X I I_{1}}(x)=k_{1} e^{\lambda x} x^{c}\left[\frac{c}{x}+\lambda\right]\left(1+e^{\lambda x} x^{c}\right)^{-k_{1}-1}, \quad x>0
$$

and $Y$ be a random variable following the $M B X I I$ distribution with parameters $c, k_{2}$ and $\lambda$, i.e., with the pdf given by

$$
f_{M B X I I_{2}}(x)=k_{2} e^{\lambda x} x^{c}\left[\frac{c}{x}+\lambda\right]\left(1+e^{\lambda x} x^{c}\right)^{-k_{2}-1}, \quad x>0 .
$$

Then, if $k_{2} \leq k_{1}$, we have $X \leq_{l r} Y$, i.e., $f_{M B X I I_{1}}(x) / f_{M B X I I_{2}}(x)$ is decreasing.

Indeed, we have

$$
\frac{f_{M B X I I_{1}}(x)}{f_{M B X I I_{2}}(x)}=\frac{k_{1}}{k_{2}}\left(1+e^{\lambda x} x^{c}\right)^{-k_{1}+k_{2}} .
$$

Taking derivative with respect to $x$ we have

$$
\frac{\partial}{\partial x} \frac{f_{M B X I I_{1}}(x)}{f_{M B X I I_{2}}(x)}=\frac{k_{1}}{k_{2}}\left(k_{2}-k_{1}\right)\left(1+e^{\lambda x} x^{c}\right)^{-k_{1}+k_{2}-1} e^{\lambda x} x^{c}\left[\frac{c}{x}+\lambda\right],
$$

Therefore, if $k_{2} \leq k_{1}$, we have $\partial f_{M B X I I_{1}}(x) / f_{M B X I I_{2}}(x) / \partial x \leq 0$, implying that the ratio function $f_{M B X I I_{1}}(x) / f_{M B X I I_{2}}(x)$ is decreasing, proving that the desired likelihood ratio ordering exists. This ends the proof of Proposition 2.

3.6. Reliability parameter. A result on the reliability parameter involving two MBXII distributions with $c$ and $\lambda$ as common parameters is given below. For the importance of this parameter in statistics, we refer to [12].

Proposition 3. Let $X$ be a random variable following the MBXII distribution with parameters $c, k_{1}$ and $\lambda$, i.e., with the pdf given by

$$
f_{M B X I I_{1}}(x)=k_{1} e^{\lambda x} x^{c}\left[\frac{c}{x}+\lambda\right]\left(1+e^{\lambda x} x^{c}\right)^{-k_{1}-1}, \quad x>0,
$$

and $Y$ be a random variable following the $M B X I I$ distribution with parameters $c, k_{2}$ and $\lambda$, i.e., with the cdf given by

$$
F_{M B X I I_{2}}(x)=1-\left(1+e^{\lambda x} x^{c}\right)^{-k_{2}}, \quad x>0 .
$$

Let $R=\mathbb{P}(X<Y)$ be the reliability parameter. Then we have

$$
R=\frac{k_{2}}{k_{1}+k_{2}} .
$$


Proof. After some algebra, we obtain

$$
\begin{aligned}
R & =\int_{-\infty}^{+\infty} f_{M B X I I_{1}}(x) F_{M B X I I_{2}}(x) d x \\
& =\int_{0}^{+\infty} k_{1} e^{\lambda x} x^{c}\left[\frac{c}{x}+\lambda\right]\left(1+e^{\lambda x} x^{c}\right)^{-k_{1}-1}\left[1-\left(1+e^{\lambda x} x^{c}\right)^{-k_{2}}\right] d x \\
& =1-\int_{0}^{+\infty} k_{1} e^{\lambda x} x^{c}\left[\frac{c}{x}+\lambda\right]\left(1+e^{\lambda x} x^{c}\right)^{-k_{1}-k_{2}-1} d x \\
& =1+\left.\frac{k_{1}}{k_{1}+k_{2}}\left(1+e^{\lambda x} x^{c}\right)^{-k_{1}-k_{2}}\right|_{0} ^{+\infty}=\frac{k_{2}}{k_{1}+k_{2}} .
\end{aligned}
$$

Proposition 3 is proved.

3.7. Order statistics. Let $X_{1}, X_{2}, \ldots, X_{n}$ be $n$ iid random variables following two MBXII distributions with pdf and cdf given by (2) and (1), respectively. By arranging them in increasing order, we can relabel these variables as $X_{1: n}<X_{2: n}<\ldots<X_{n: n}$. A result related to the $i^{\text {th }}$ order statistic $X_{i: n}$ is presented below.

Proposition 4. The pdf of $X_{i: n}$ can be expressed as a linear combination of $i$ pdfs of the MBXII distribution.

Proof. A well-known result ensures that the pdf of $X_{i: n}$ is given by

$$
f_{i: n}(x)=\frac{n !}{(i-1) !(n-i) !} f_{M B X I I}(x)\left[F_{M B X I I}(x)\right]^{i-1}\left[1-F_{M B X I I}(x)\right]^{n-i} .
$$

The binomial formula gives

$$
\left[F_{M B X I I}(x)\right]^{i-1}=\left[1-\left(1+e^{\lambda x} x^{c}\right)^{-k}\right]^{i-1}=\sum_{j=0}^{i-1}\left(\begin{array}{c}
i-1 \\
j
\end{array}\right)(-1)^{j}\left(1+e^{\lambda x} x^{c}\right)^{-k j}
$$

Hence, by using the expression of $f_{M B X I I}(x)$, we obtain

$$
\begin{aligned}
& f_{i: n}(x) \\
& =\frac{n !}{(i-1) !(n-i) !} \sum_{j=0}^{i-1}\left(\begin{array}{c}
i-1 \\
j
\end{array}\right)(-1)^{j}\left\{f_{M B X I I}(x)\left(1+e^{\lambda x} x^{c}\right)^{-k j-k(n-i)}\right\} \\
& =\frac{n !}{(i-1) !(n-i) !} \sum_{j=0}^{i-1}\left(\begin{array}{c}
i-1 \\
j
\end{array}\right)(-1)^{j} f_{i, j, n}^{*}(x),
\end{aligned}
$$

where

$$
f_{i, j, n}^{*}(x)=k e^{\lambda x} x^{c}\left[\frac{c}{x}+\lambda\right]\left(1+e^{\lambda x} x^{c}\right)^{-k(n+j-i+1)-1}, \quad x>0 .
$$

We end the proof of Proposition 4 by noticing that $f_{i, j, n}^{*}(x)$ is the pdf of the MBXII distribution with parameters $c, \lambda$ and $k(n+j-i+1)$. 
By virtue of Proposition 4, we can express the $r^{\text {th }}$ moment for $X_{i: n}$ according to the $r^{\text {th }}$ moment of the MBXII distribution with parameters $c, \lambda$ and $k(n+j-i+1)$, which can be determined by using the results in Subsection 3.2. More precisely, by using the expression (5), we have

$$
\mathbb{E}\left(X_{i: n}^{r}\right)=\frac{n !}{(i-1) !(n-i) !} \sum_{j=0}^{i-1}\left(\begin{array}{c}
i-1 \\
j
\end{array}\right)(-1)^{j} \int_{-\infty}^{+\infty} x^{r} f_{i, j, n}^{*}(x) d x .
$$

Then we can derive mean of $X_{i: n}$, variance, skewness, kurtosis...

\section{Statistical inference}

4.1. Estimation. We now investigate the estimation of the MBXII model with parameters $c, k$ and $\lambda$. Let $x_{1}, x_{2}, \ldots, x_{n}$ be a random sample of size $n$ from the MBXII distribution with pdf given by (2). Then the likelihood function for the vector of parameters $\Theta=(c, k, \lambda)^{T}$ is given by

$$
\begin{aligned}
\ell & =\sum_{i=1}^{n} \log \left[f_{M B X I I}\left(x_{i}\right)\right] \\
& =n \log (k)+c \sum_{i=1}^{n} \log \left(x_{i}\right)+\lambda \sum_{i=1}^{n} x_{i}+\sum_{i=1}^{n} \log \left(\frac{c}{x_{i}}+\lambda\right) \\
& -(k+1) \sum_{i=1}^{n} \log \left(1+e^{\lambda x_{i}} x_{i}^{c}\right) .
\end{aligned}
$$

The components of score vector $\Theta=(c, k, \lambda)^{T}$ are

$$
\begin{aligned}
& \frac{\partial \ell}{\partial k}=\frac{n}{k}-\sum_{i=1}^{n} \log \left(1+e^{\lambda x_{i}} x_{i}^{c}\right), \\
& \frac{\partial \ell}{\partial c}=\sum_{i=1}^{n} \log \left(x_{i}\right)+\sum_{i=1}^{n}\left[\frac{1}{c+\lambda x_{i}}\right]-(k+1) \sum_{i=1}^{n}\left[\frac{e^{\lambda x_{i}} x_{i}^{c} \log \left(x_{i}\right)}{1+e^{\lambda x_{i}} x_{i}^{c}}\right], \\
& \frac{\partial \ell}{\partial \lambda}=\sum_{i=1}^{n} x_{i}+\sum_{i=1}^{n}\left[\frac{x_{i}}{c+\lambda x_{i}}\right]-(k+1) \sum_{i=1}^{n}\left[\frac{x_{i}^{c+1} e^{\lambda x_{i}}}{1+e^{\lambda x_{i}} x_{i}^{c}}\right] .
\end{aligned}
$$

Solving these equations simultaneously yields the maximum likelihood estimates (MLEs) of the three parameters. Numerical iterative techniques are then necessary to estimate the model parameters. It is possible to determine the global maxima of the log-likelihood by taking different initial values for the parameters. However, we observed that the MLEs for this model is not very sensitive to the initial estimates. For interval estimation on the model parameters, we require the Fisher information matrix; however in this article we leave this routine calculation to an interested reader. 
4.2. Simulation study. In this section, the performance of the MLEs of the MBXII parameters are discussed by means of Monte-Carlo simulation study. The following measures are used to evaluate the simulation results: bias, mean square error (MSE), estimated average length (AL) and coverage probability (CP). The $N=1000$ samples of sizes $n=50,55, \ldots, 1000$ are generated from the MBXII distribution with the parameters values: $k=2, c=2, \lambda=2$, by using the inverse transform method. The MLEs of the parameters of MBXII distribution are obtained for each generated sample, $\left(\hat{k}_{i}, \hat{c_{i}}, \hat{\lambda_{i}}\right)$. The standard errors of the MLEs are obtained by inverting the observed information matrix, $\left(s_{\hat{k_{i}}}, s_{\hat{c_{i}}}, s_{\hat{\lambda_{i}}}\right)$. The formulas for biases, MSEs, CPs and ALs can be found in [2].

Figure 5 shows the results for simulation plan given above. We can conclude that the estimated biases are positive for all parameters. The estimated biases decrease as the sample size $n$ increases. Further, the estimated MSEs are so closed to zero for large sample sizes. This result reveals the consistency property of the MLEs. The CP approaches to the nominal value (0.95) when the sample size increases. It is clear that when the sample size increases, the AL decreases for all parameters. 

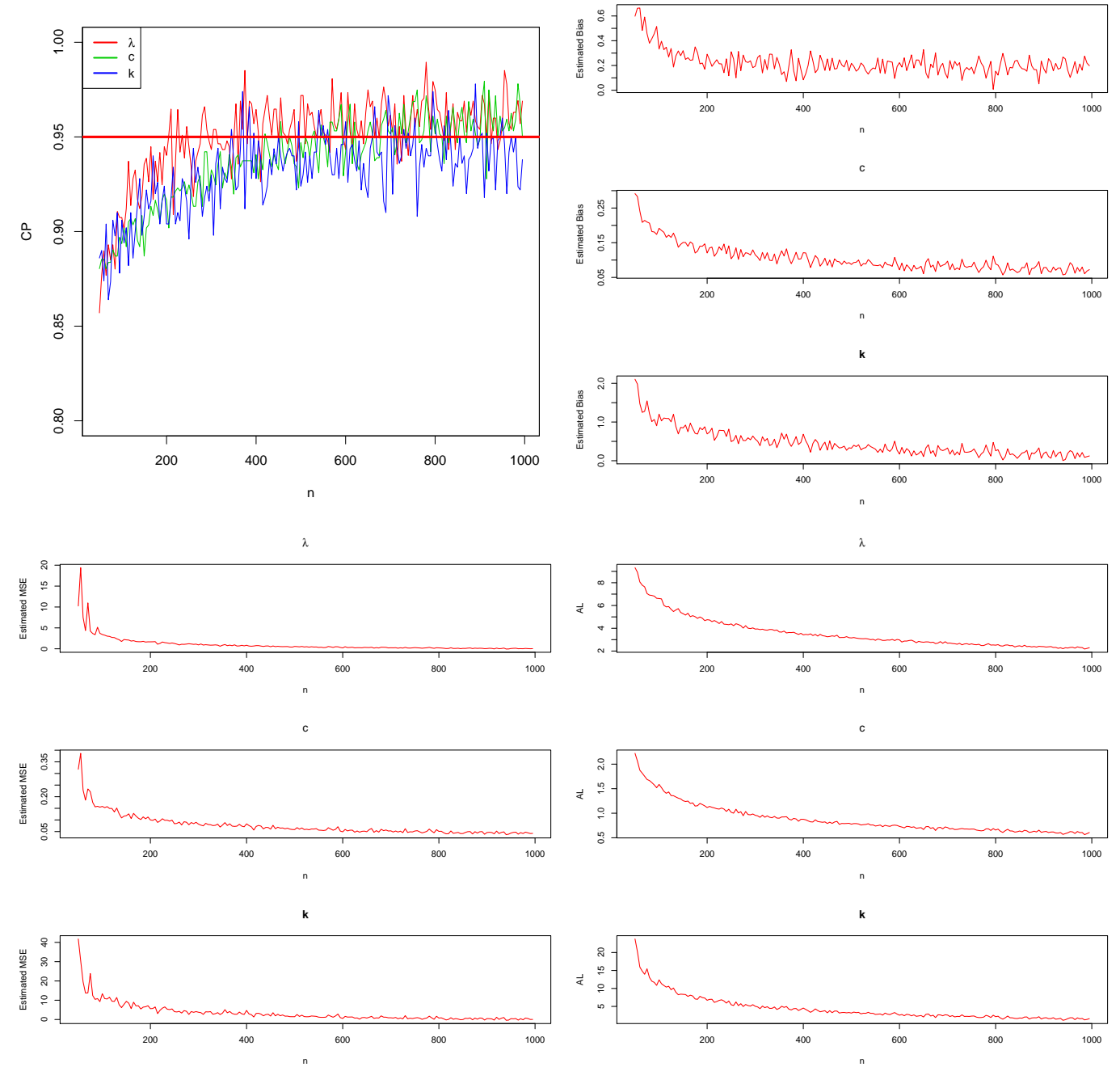

Figure 5. Estimated CPs, biases, MSEs and ALs for the selected parameters. 


\section{Applications}

This section provides three applications to show how the MBXII distribution can be applied in practice. We aim to compare the MBXII model to two natural competitors: the new Burr XII (NBXII) (see [26]) and the former Burr XII (BXII) models, i.e., with respective cdfs given by

$$
\begin{aligned}
F_{N B X I I}(x) & =\left[1+e^{-\left(\frac{x-\mu}{\sigma}\right)^{3}}\right]^{-p}, \quad \sigma, p>0, x, \mu \in \mathbb{R} \\
F_{B X I I}(x) & =1-\left(1+x^{c}\right)^{-k}, \quad x, c, k>0 .
\end{aligned}
$$

The details of our coming methodology are the following. The MLEs are computed using Quasi-Newton Code for Bound Constrained Optimization (also known as L-BFGS-B). Then, it is use to determine the confidence intervals (CI) of the MBXII parameters and the log-likelihood function evaluated $(\ell)$. The goodness-of-fit measures, Anderson-Darling $\left(A^{*}\right)$, Cramer-von Mises $\left(W^{*}\right)$ are computed. As usual, the lower the values of these criteria, the better the fit. The value for the Kolmogorov Smirnov $(K S)$ statistic and its p-value are also provided. The definitions of these measures can be found in [8]. The plots of the fitted pdfs and cdfs of some distributions are displayed for visual comparison. The required computations are carried out in the $R$ software.

\section{Data set 1}

The first data is taken from [18]. It represents 20 observations on failure of components. Table 3 presents the basic statistical measures of Data set 1.

TABle 3. Descriptive Statistics for Data set 1.

\begin{tabular}{ccccccc}
\hline & $n$ & Mean & Median & Variance & skewness & kurtosis \\
\hline Data Set 1 & 20 & 0.1612 & 0.1328 & 0.0247 & 1.3302 & 1.5144 \\
\hline
\end{tabular}

Figure 6 complete the descriptive statistics by showing the box plot of Data set 1, as well as the TTT-plot which indicates a possible near constant or unimodal hrf. The MBXII hrf can have this property.

\section{Data set 2}

The second data is taken from [17]. It represents the failure times of 50 components (per 1000h). A description of the basics statistics for Data set 2 is done in Table 4.

TABle 4. Descriptive Statistics for Data set 2.

\begin{tabular}{ccccccc}
\hline & $n$ & Mean & Median & Variance & skewness & kurtosis \\
\hline Data Set 2 & 50 & 3.3429 & 1.4140 & 17.4847 & 1.4167 & 1.0846 \\
\hline
\end{tabular}


Figure 7 shows the box plot of Data set 2, as well as the TTT-plot. The convexity of the curve indicates a possible near monotonically increasing or unimodal hrf. The MBXII distribtion covers this case.

\section{Data set 3}

The third data set is taken from [21]. It is the 50 observations on Burr (in the unit of millimeter), the hole diameter is $12 \mathrm{~mm}$ and the sheet thickness is $3.15 \mathrm{~mm}$. Table 5 presents the basic statistical measures of Data set 1 .

TABle 5. Descriptive Statistics for Data set 3.

\begin{tabular}{ccccccc}
\hline & $n$ & Mean & Median & Variance & skewness & kurtosis \\
\hline Data Set 3 & 50 & 0.1632 & 0.1600 & 0.0065 & 0.0723 & -0.7833 \\
\hline
\end{tabular}

Figure 8 shows the box plot of Data set 3, as well as the TTT-plot. The concavity of the curve indicates a possible near monotonically decreasing hrf. The MBXII hrf can have this property.

Hence, Tables 6, 9 and 12 indicate the MLEs for the models parameters (with their standard errors for Data sets 1,2 and 3, respectively. Tables 7, 10 and 13 indicate the CIs for the MBXII parameters at the levels $95 \%$ and $99 \%$. Tables 8,11 and 14 show the obtained values for $\ell, W^{*}, A^{*}, K S$ and p-value for Data sets 1, 2 and 3, respectively. All the numerical results can be summarized in one sentence: the MBXII model presents the best fits for the three data sets. Finally, Figures 9, 10 and 11 show fitted pdfs and cdfs of the considered models for Data sets 1, 2 and 3, respectively. Thus, the nice fitting of the MBXII is observed.

TABLE 6. MLEs for the models parameters and their standard errors (in parentheses) for Data set 1.

\begin{tabular}{ccccccc}
\hline Distribution & $c$ & $k$ & $\lambda$ & $\mu$ & $\sigma$ & $p$ \\
\hline MBXII & 0.6412 & 2.1195 & 3.5003 & - & - & - \\
& $(0.2816)$ & $(0.1643)$ & $(1.1021)$ & - & - & - \\
NBXII & - & - & - & -1.7931 & 1.0822 & 215.5150 \\
& - & - & - & $(0.6365)$ & $(0.2693)$ & $(373.9932)$ \\
BXII & 0.9671 & 6.7647 & - & - & - & - \\
& $(0.1654)$ & $(2.1224)$ & - & - & - & - \\
\hline
\end{tabular}


TABLE 7. Confidence intervals of the MBXII parameters for Data set 1.

\begin{tabular}{|c|c|c|c|}
\hline $\mathrm{CI}$ & $c$ & $k$ & $\lambda$ \\
\hline $95 \%$ & {$\left[\begin{array}{lll}0.0892 & 1.0061\end{array}\right]$} & {$\left[\begin{array}{lll}1.7974 & 2.4415\end{array}\right]$} & {$\left[\begin{array}{ll}1.3401 & 5.6604\end{array}\right]$} \\
\hline $99 \%$ & {$\left[\begin{array}{ll}0 & 1.3677\end{array}\right]$} & {$\left[\begin{array}{lll}1.6956 & 2.5433\end{array}\right]$} & {$\left[\begin{array}{lll}0.6568 & 6.3437\end{array}\right]$} \\
\hline
\end{tabular}

TABLE 8. The $\ell, W^{*}, A^{*}, K S$, p-value values for Data set 1.

\begin{tabular}{cccccc}
\hline Distribution & $\ell$ & $W^{*}$ & $A^{*}$ & $K S$ & $\mathrm{p}$-value \\
\hline MBXII & -17.3934 & 0.0188 & 0.1209 & 0.0869 & 0.9958 \\
NBXII & -11.4799 & 0.0597 & 0.4594 & 0.1421 & 0.7629 \\
BXII & -16.2927 & 0.0521 & 0.2968 & 0.1278 & 0.8588 \\
\hline
\end{tabular}

TABLE 9. MLEs for the models parameters and their standard errors (in parentheses) for Data set 2 .

\begin{tabular}{ccccccc}
\hline Distribution & $c$ & $k$ & $\lambda$ & $\mu$ & $\sigma$ & $p$ \\
\hline MBXII & 0.7110 & 0.7202 & 0.1726 & - & - & - \\
& $(0.1270)$ & $(0.1632)$ & $(0.0880)$ & - & - & - \\
NBXII & - & - & - & 17.5624 & 2.5776 & 0.0049 \\
& - & - & - & $(1.0120)$ & $(0.4766)$ & $(0.0024)$ \\
BXII & 0.9091 & 0.9943 & - & - & - & - \\
& $(0.1133)$ & $(0.1549)$ & - & - & - & - \\
\hline
\end{tabular}

TABLE 10. Confidence intervals of the MBXII parameters for Data set 2.

\begin{tabular}{|c|c|c|c|}
\hline $\mathrm{CI}$ & $c$ & $k$ & $\lambda$ \\
\hline $95 \%$ & {$\left[\begin{array}{lll}0.4620 & 0.9599\end{array}\right]$} & {$\left[\begin{array}{lll}0.4003 & 1.0400\end{array}\right]$} & {$\left[\begin{array}{lll}0.0001 & 0.3450\end{array}\right]$} \\
\hline $99 \%$ & {$\left[\begin{array}{lll}0.3833 & 1.0386\end{array}\right]$} & {$\left[\begin{array}{lll}0.2991 & 1.1412\end{array}\right]$} & {$\left[\begin{array}{ll}0 & 0.3996\end{array}\right]$} \\
\hline
\end{tabular}




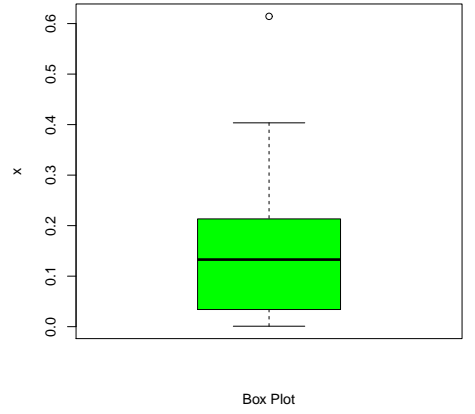

(a)

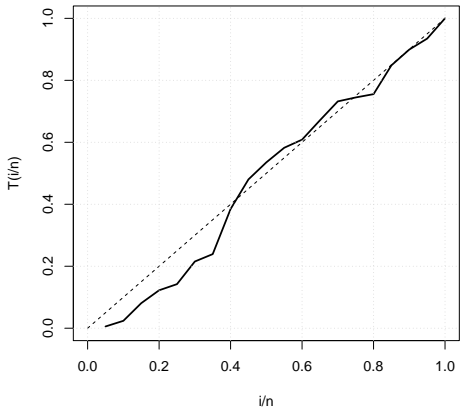

(b)

Figure 6. Graphics of (a) Box plot (b) TTT-plot for Data set 1.

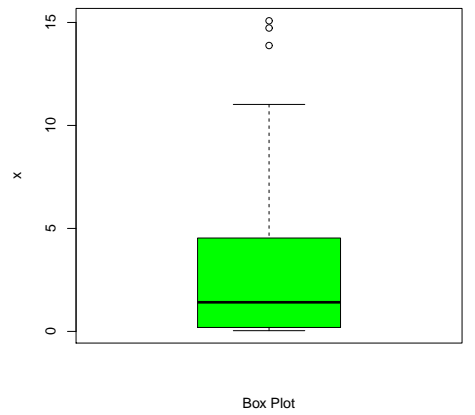

(a)

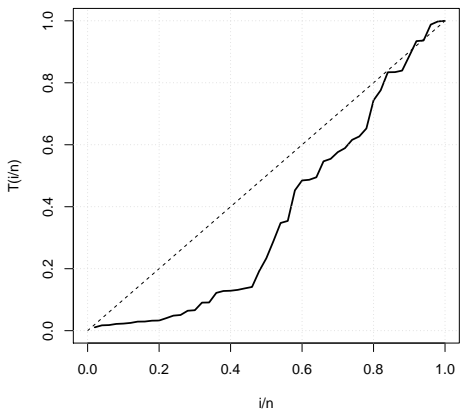

(b)

Figure 7. Graphics of (a) Box plot (b) TTT-plot for Data set 2.

TABle 11. The $\ell, W^{*}, A^{*}, K S, \mathrm{p}$-value values for Data set 2 .

\begin{tabular}{cccccc}
\hline Distribution & $\ell$ & $W^{*}$ & $A^{*}$ & $K S$ & p-value \\
\hline MBXII & 101.3061 & 0.1337 & 0.8456 & 0.1100 & 0.5432 \\
NBXII & 145.8011 & 0.7372 & 4.2634 & 0.2324 & 0.0073 \\
BXII & 105.5833 & 0.2158 & 1.3411 & 0.15657 & 0.1547 \\
\hline
\end{tabular}




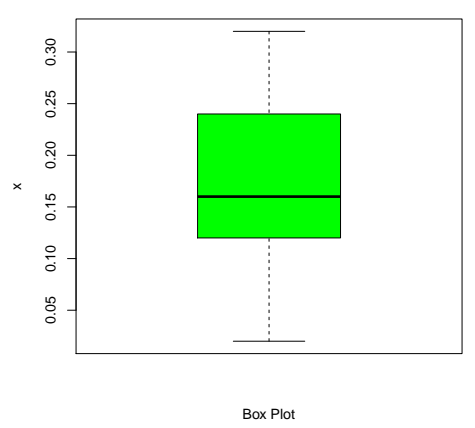

(a)

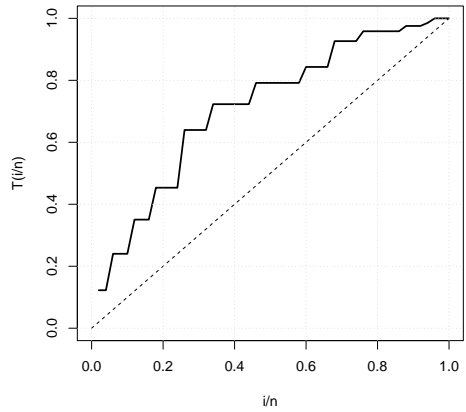

(b)

Figure 8. Graphics of (a) Box plot (b) TTT-plot for Data set 3.

TABLE 12. MLEs for the models parameters and their standard errors (in parentheses) for Data set 3 .

\begin{tabular}{ccccccc}
\hline Distribution & $c$ & $k$ & $\lambda$ & $\mu$ & $\sigma$ & $p$ \\
\hline MBXII & 1.5714 & 6.4284 & 4.5271 & - & - & - \\
& $(0.4101)$ & $(2.0121)$ & $(0.6087)$ & - & - & - \\
NBXII & - & - & - & -0.5562 & 0.4706 & 23.4050 \\
& - & - & - & $(0.3068)$ & $(0.1438)$ & $(30.0250)$ \\
BXII & 2.1543 & 39.0950 & - & - & - & - \\
& $(0.2401)$ & $(14.9952)$ & - & - & - & - \\
\hline
\end{tabular}

TABLE 13. Confidence intervals of the MBXII parameters for Data set 3.

\begin{tabular}{|c|c|c|c|}
\hline CI & $c$ & $k$ & $\lambda$ \\
\hline $95 \%$ & {$\left[\begin{array}{lll}0.7676 & 2.3751\end{array}\right]$} & {$\left[\begin{array}{lll}2.4846 & 10.3721\end{array}\right]$} & {$\left[\begin{array}{lll}3.3354 & 5.7187\end{array}\right]$} \\
\hline $99 \%$ & {$\left[\begin{array}{lll}0.5133 & 2.6294\end{array}\right]$} & {$\left[\begin{array}{lll}1.2371 & 11.6196\end{array}\right]$} & {$\left[\begin{array}{lll}2.9584 & 6.0957\end{array}\right]$} \\
\hline
\end{tabular}


TABLE 14 . The $\ell, W^{*}, A^{*}, K S, \mathrm{p}$-value values for Data set 3 .

\begin{tabular}{cccccc}
\hline Distribution & $\ell$ & $W^{*}$ & $A^{*}$ & $K S$ & $\mathrm{p}$-value \\
\hline MBXII & -56.6981 & 0.07991 & 0.5011 & 0.1009 & 0.6782 \\
NBXII & -54.8154 & 0.0868 & 0.5734 & 0.1091 & 0.5910 \\
BXII & -55.7357 & 0.1078 & 0.6605 & 0.1101 & 0.5794 \\
\hline
\end{tabular}
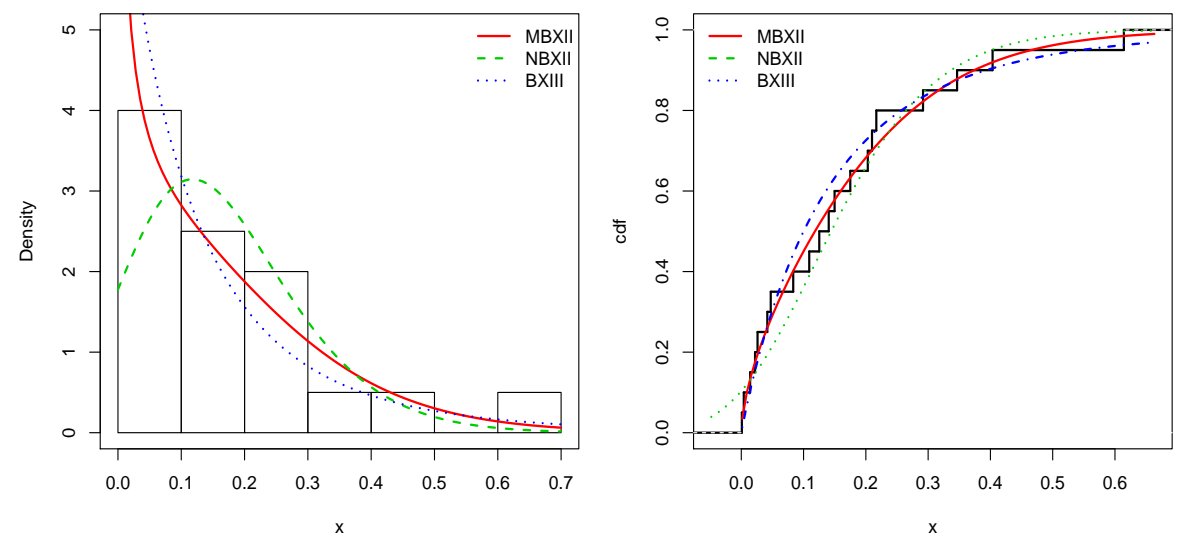

Figure 9. Estimated pdfs and cdfs for Data set 1. 

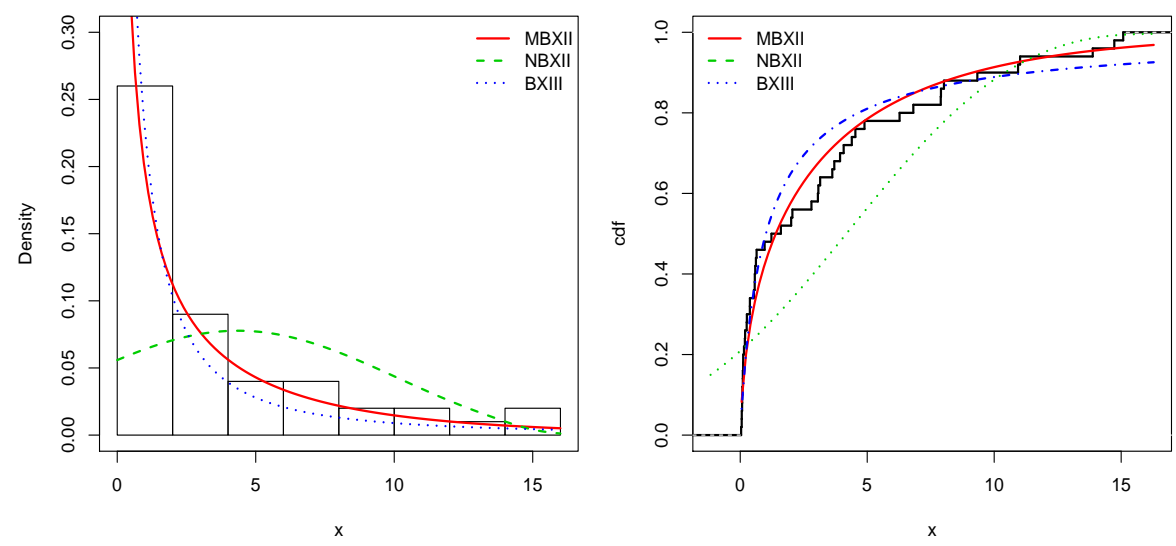

Figure 10. Estimated pdfs and cdfs for Data set 2.
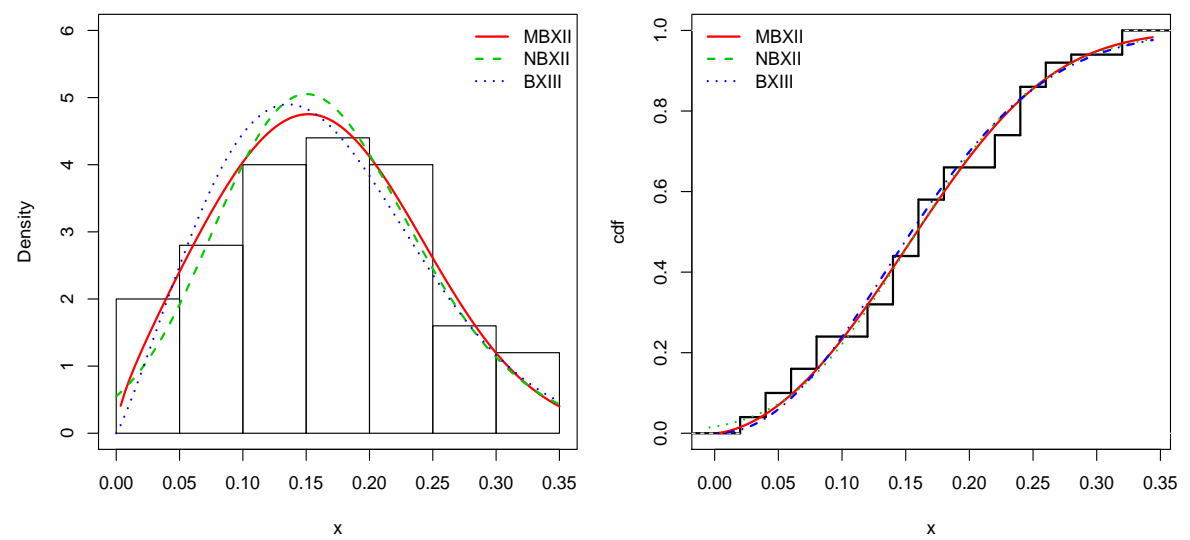

Figure 11. Estimated pdfs and cdfs for Data set 3. 


\section{Concluding Remarks}

A growing interest among the statisticians is to construct the more flexible lifetime model possible. In particular, several authors proposed new distributions that are based on the traditional Burr model. In this paper, we offered a promising one by introducing the MBXII distribution. Their mathematical properties are discussed as shapes, asymptotes, useful expansion, quantiles, moments, skewness, kurtosis, incomplete moments, moment generating function, stochastic ordering, reliability parameter and order statistics. Then, we explored the MBXII parametric model, with estimation of the parameters via the maximum likelihood method. The validity of this method was confirmed by a simulation study. The proposed distribution was utilized to model four data sets; it was shown to provide a better fit than other related models. The distributional results developed in this article should find numerous applications in reliability theory, hydrology, medicine, meteorology, survival analysis and engineering.

\section{REFERENCES}

[1] Abdel-Hamid, A. H. (2009). Constant-Partially Accelerated Life Tests for Burr XII Distribution with Progressive Type II Censoring, Computational Statistics and Data Analysis, 53, 7, 2511-2523.

[2] Afify, A. Z., Altun, E., Alizadeh, M., Ozel, G. and Hamedani, G. G. (2017). The odd exponentiated half-logistic-G family: properties, characterizations and applications. Chilean Journal of Statistics, 8, 65-91.

[3] Afify, A. Z., Cordeiro, G. M., Ortega, E. M. M., Yousof, H. M. and Butt, N. S. (2016). The fourparameter Burr XII distribution: properties, regression model and applications. Communications in Statistics-Theory and Methods, DOI: 10.1080/03610926.2016.1231821.

[4] Afify, A. Z., Cordeiro, G. M., Bourguignon, M. and Ortega, E. M. M. (2018). Properties of the Transmuted Burr XII Distribution, Regression and its Applications, Journal of Data Science, 16, 3, 485-510.

[5] AL-Hussaini, E. K., Mousa, M. A. and Jaheen, Z. F. (1992). Estimation under the Burr Type XII Failure Model: A Comparative Study, Test, 1, 1, 33-42.

[6] Al-Saiari, A. Y., Baharith, L. A. and Mousa, S. A. (2014). Marshall-Olkin extended Burr type XII distribution. International Journal of Statistics and Probability, 3, 78-84.

[7] Burr, I. W. (1942). Cumulative frequency distributions. Ann. Math. Stat., 13, 215-232.

[8] Chen, G. and Balakrishnan, N. (1995). A general purpose approximate goodness-of-fit test, Journal of Quality Technology, 27, 154-161.

[9] Decani, J. S. and Stine, R. A. (1986). A note on deriving the information matrix for a logistic distribution. The American Statistician. American Statistical Association, 40, 220-222.

[10] Drane, S. W., Owen, D. B. and Seibetr Jr., G. B. (1978). The Burr Distribution and Quantal Responses, Statistical Papers, 19, 3, 204-210.

[11] Gomes, E. E., da-Silva, C. Q. and Cordeiro, G. M. (2015). Two extended Burr models: Theory and practice. Communication in Statistics Theory-Methods, 44, 1706-1734.

[12] Kotz, S., Lumelskii, Y. and Penskey, M. (2003). The stress-strength model and its generalizations and applications, World Scientific, Singapore.

[13] Lai, C. D., Xie, M., and Murthy, D. N. P. (2003). A modified Weibull distribution. IEEE Transactions on Reliability, 52, 33-37.

[14] Lomax, K. S. (1954). Business Failures; Another example of the analysis of failure data. Journal of the American Statistical Association, 49, 847-852. 
[15] Moore, D. and Papadopoulos, A. S. (2000). The Burr Type XII Distribution as a Failure Model under Various Loss Functions, Microelectronics Reliability, 40, 12, 2117-2122.

[16] Muhammad, M. (2016), A generalization of the Burr XII-Poisson distribution and its applications. Journal of Statistics Applications and Probability, 5, 1, 29 - 41.

[17] Murthy, D. P., Xie, M., and Jiang, R. (2004). Weibull models (Vol. 505). John Wiley and Sons.

[18] Nigm, A. M., AL-Hussaini, E. K. and Jaheen, Z. F. (2003). Bayesian onesample prediction of future observations under Pareto distribution. Statistics, 37, 6, 527-536.

[19] Papadopoulos, A. S. (1978). The Burr Distribution as a Life Time Model from a Bayesian Approach, IEEE Transactions on Reliability, 27, 5, 369-371.

[20] Paranaíba, P. F., Ortega, E. M. M., Cordeiro, G. M. and Pescim, R. R. (2011). The beta Burr XII distribution with application to lifetime data. Computation Statistics and Data Analysis, 55, 11181136.

[21] Ratan, D. (2011). On the distribution of Burr with applications, Sankhya, 73, 1-19.

[22] Rodriguez, R. N. (1977). A Guide to the Burr Type XII Distributions, Biometrika, 64, 1, $29-134$.

[23] Shaked, M. and Shanthikumar, J. G. (1994). Stochastic orders and their applications. Academic Press, New York.

[24] Shoukri, M.M., Mian, I.U.H. and Tracy, D.S. (1988). Sampling properties of estimators of the loglogistic distribution with application to Canadian precipitation data. The Cand. J. Stat., 16, 3, 223236.

[25] Tadikamalla, P. R. (1980). A Look at the Burr and Related Distributions, International Statistical Review, 48, 3, 37-344.

[26] Yari, G. and Tondpour, Z. (2017). The new Burr distribution and its application, Math Sci, 11, 47-54. 